\title{
Points de vue d'actualité : violences obstétricales
}

\section{Breaking News: Gynaecological Violences}

\section{Hernandorena}

(C) Lavoisier SAS 2017

Depuis le mois d'août dernier, des medias se sont fait l'écho de paroles de femmes témoignant de violences obstétricales inacceptables.

Bien sûr, les professionnels peuvent se sentir injustement blessés, mais nous devons prendre ces terribles paroles qui témoignent d'une réalité comme une opportunité et c'est l'ensemble de la périnatalogie qui doit se sentir concernée par le vécu de ces femmes et qui doit réagir.

Ces paroles nous incitent à poursuivre et consolider les améliorations survenues dans la qualité des prises en charge dans le domaine de la périnatalité. En effet, depuis de nombreuses années, les sociétés savantes et particulièrement la SFMP, par son côté multidisciplinaire, ont beaucoup fait progresser tous les aspects notamment psychologiques de nos pratiques. Ainsi, la très grande majorité des unités d'obstétrique travaillent très bien, en conjuguant sécurité émotionnelle et qualité scientifique. Et la majorité des femmes sont satisfaites de leur prise en charge.

Mais nous ne devons pas nous en satisfaire et ces paroles sont une excellente occasion pour continuer à se remettre en question, vérifier les fonctionnements des unités en mettant en avant les pratiques transversales, fondements de la périnatalogie et également repenser la formation des internes en obstétrique au niveau des services, la formation des sagesfemmes dans les écoles et les unités.

Je veux rajouter une vision néonatale à cette problématique. Nous avons également énormément progressé dans la prise en charge des nouveau-nés et de leur famille. Mais il peut exister aussi les situations de violences indirectes faites aux parents dont les conséquences sur leur lien avec l'enfant peuvent être dramatiques pour son avenir, à partir des mêmes causes : absence de mots, de dialogue, ou paroles déplacées, absence de regard, ou mauvais regard, absence d'écoute. Ce sont toujours ces remarques qui sont souvent décrites négativement par le retour des parents. La prise en compte émotionnelle passe par la conscience du poids des mots, des regards, des postures. Les soins de développement en néonatalogie vont dans ce sens. Il faut poursuivre leur implantation.

Notre revue a donc demandé à plusieurs personnalités venant d'horizons très différents mais unies par l'esprit de la médecine périnatale de réagir à ces témoignages de situations de violences obstétricales : Mme Phan, représentant les usagers au sein du collectif CIANE; Mme Mulin et Mr Burguet, représentant la Fédération des Réseaux en Périnatalité ; Mr D’Ercole, Président sortant de la SFMP ; Mmes Yamgnane et Molénat avec la Société de psychologie périnatale.

Par ailleurs, en élargissant la problématique des violences faites aux femmes, la revue tient à saluer la naissance d'un nouveau diplôme interuniversitaire autour des Facultés de Paris Descartes, Montpellier et Grenoble, à l'initiative d'un groupe pluridisciplinaire, diplôme qui prendra en compte les aspects obstétricaux : «Prise en charge des violences faites aux femmes, vers la bientraitance », dont voici le lien : Dr Perrine Millet, Présidente (unmaillonmanquant.org).

$\mathrm{X}$. Hernandorena $(\bowtie)$

1780 route de l'Aqueduc, 13990 Fontvieille, France

e-mail : xavier.hernandorena@orange.fr 